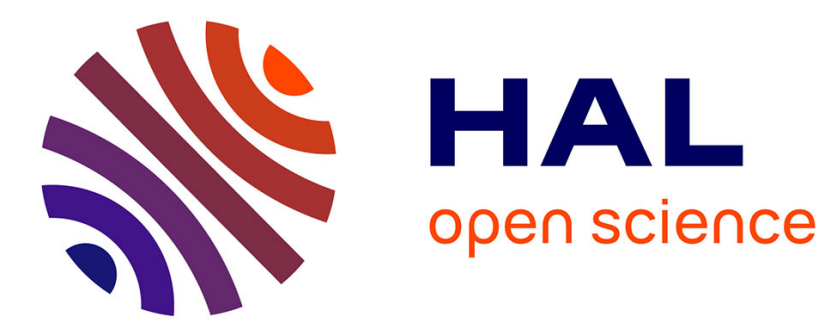

\title{
High-pressure phase transition in gallium phosphide: An x-ray-absorption spectroscopy study
}

\author{
J.P. Itié, A. Polian, C Jauberthie-Carillon, E Dartyge, A Fontaine, H \\ Tolentino, G Tourillon
}

\section{- To cite this version:}

J.P. Itié, A. Polian, C Jauberthie-Carillon, E Dartyge, A Fontaine, et al.. High-pressure phase transition in gallium phosphide: An x-ray-absorption spectroscopy study. Physical Review B: Condensed Matter (1978-1997), 1989. hal-03059442

\section{HAL Id: hal-03059442 \\ https://hal.science/hal-03059442}

Submitted on 12 Dec 2020

HAL is a multi-disciplinary open access archive for the deposit and dissemination of scientific research documents, whether they are published or not. The documents may come from teaching and research institutions in France or abroad, or from public or private research centers.
L'archive ouverte pluridisciplinaire HAL, est destinée au dépôt et à la diffusion de documents scientifiques de niveau recherche, publiés ou non, émanant des établissements d'enseignement et de recherche français ou étrangers, des laboratoires publics ou privés. 


\title{
High-pressure phase transition in gallium phosphide: An x-ray-absorption spectroscopy study
}

\author{
J. P. Itie, A. Polian, and C. Jauberthie-Carillon \\ Physique des Milieux Condensés, Université Pierre et Marie Curie, Tour 13-4e etage, \\ 4 place Jussieu, F-75252 Paris CEDEX 05, France \\ E. Dartyge, A. Fontaine, H. Tolentino, and G. Tourillon \\ Laboratoire pour l'Utilisation du Rayonnement Electromagnétique, Université de Paris-Sud, \\ Bâtiment 209D, F-91405 Orsay CEDEX, France \\ (Received 6 February 1989)
}

\begin{abstract}
High-pressure behavior of GaP has been studied by $\mathrm{x}$-ray-absorption spectroscopy in a diamondanvil cell up to $36 \mathrm{GPa}$. The room-temperature equation of state of phase $\mathrm{I}$ has been determined. The transition from a fourfold (zinc-blende) to a sixfold ( $\beta$-tin) coordination scheme is observed near $26 \mathrm{GPa}$. Analysis of the data obtained on a sample quickly depressurized from $36 \mathrm{GPa}$ shows that the transition is not reversible and the recovered sample is mainly amorphous.
\end{abstract}

\section{INTRODUCTION}

Gallium phosphide is a semiconductor which crystallizes in the zinc-blende structure ( $T_{d}^{2}$ space group). In this structure, each atom is tetrahedrally bonded to its first neighbors. Under pressure, a transition to a metallic centered tetragonal phase $(\beta$-tin) has been shown to occur in the 21-25 GPa range.

High-pressure properties of the zinc-blende structure (phase I) have been determined by Raman scattering, ${ }^{1-5}$ ultrasonic measurements, ${ }^{6}$ fundamental absorption, ${ }^{7,8}$ and refractive-index measurements. ${ }^{9}$ The transition to the metallic phase has been first observed by resistivity measurements ${ }^{10,11}$ and then by $x$-ray diffraction. ${ }^{12-14}$

However, in spite of the number of studies performed on this compound, there are still some unknowns and controversies. In particular, even the room-temperature equation of state of the zinc-blende phase has not yet been determined, and the reversibility of the transition is still questioned. $4,8,7,14$

It has been shown that $\mathrm{x}$-ray-absorption spectroscopy (XAS) and especially extended X-ray-absorption finestructure spectroscopy (EXAFS) is sensitive to pressure and gives information about the local environment of an atom: interatomic distances, number of neighbors in a shell, and the Debye-Waller factor. ${ }^{15-20}$

In the present work we have studied the high-pressure behavior of GaP up to $36 \mathrm{GPa}$ at room temperature by energy-dispersive XAS in a diamond-anvil cell (DAC) at the $K$ edge of gallium (10367 eV). This experiment enabled us to determine the equation of state of phase I $(P<25 \mathrm{GPa})$, and shows that at the transition, the gallium coordination goes from a fourfold (tetrahedral) to a sixfold (octahedral-like) environment, results which are consistent with the $\beta$-tin-like structure. Finally, analysis of the results obtained on a sample quickly depressurized from 36 to $0 \mathrm{GPa}$ shows that the transition is irreversible, the recovered sample being amorphous.
Section II of this paper is devoted to a brief presentation of the experimental techniques used, namely the energy-dispersive $\mathrm{x}$-ray-absorption spectrometer, as well as the physical parameters which can be determined by this technique. The results obtained on GaP are presented and discussed in Sec. III.

\section{EXPERIMENT}

The experiments were performed at the energydispersive X-ray-absorption port of Laboratoire pour l' Utilisation du Rayonnement Électromagnétique (LURE) (Orsay, France). The main characteristics of the spectrometer have been described elsewhere. ${ }^{21-22}$ The highpressure cell was of Block-Piermarini type. ${ }^{23}$ Several runs have been performed on fine-grained powder with silicon oil as a pressure-transmitting medium, or single crystals with the 4:1 methanol-ethanol mixture as a pressure-transmitting medium. The diameter of the experimental chamber (the hole drilled in the stainless-steel gasket) was $200 \mu \mathrm{m}$ in diameter and the initial thickness $50 \mu \mathrm{m}$. The pressure was measured outside the hutch, using the power five-ruby fluorescence scale. ${ }^{24}$

The energy dispersive scheme is particularly suited for performing x-ray-absorption spectroscopy with a classical DAC, i.e., measuring the transmission spectrum through the two anvils, for the following reasons: (i) With the new design, which utilizes an elliptically bent silicon crys$\mathrm{tal}^{22}$ the beam can be focused down to $\sim 400 \mu \mathrm{m}$ in the horizontal plane (the vertical direction is only slitted). The homogeneity of the energy distribution inside the polychromatic image of the source is almost perfect. With this geometry, an energy range of $\sim 400 \mathrm{eV}$ above the edge was accessible at the Ga $K$ edge (Fig. 1). (ii) The whole spectrum is obtained at once in a short time $(\sim 1$ s), which makes the alignment of the DAC $(\sim 200-\mu \mathrm{m}$ hole) easy, by looking at the video signal of the spectrum on an oscilloscope. (iii) Since there is no mechanical 


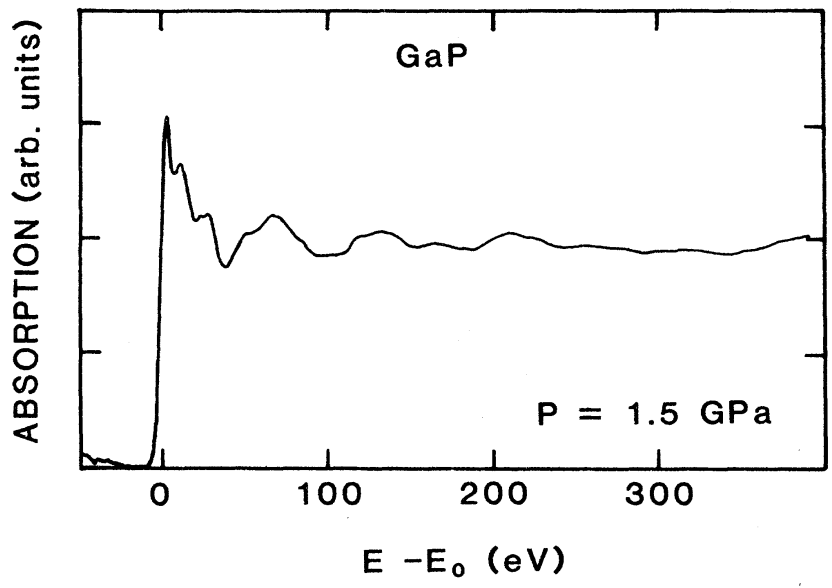

FIG. 1. X-ray absorption spectrum of $\mathrm{GaP}$ at the gallium $\mathrm{K}$ edge at $1.5 \mathrm{GPa}$.

movement of the setup, there is no instability of the energy scale or disalignment of the beam with respect to the aperture in the gasket: this last point is crucial for small samples.

As already mentioned, the x-ray beam goes through the diamond anvils and the geometry allows the use of any type of DAC. Nevertheless, it has the drawback that the Bragg conditions are fulfilled for the monocrystalline diamond anvils for specific energies, resulting in a "hijacking" of these photons. These missing photons give rise to extra absorption peaks (glitches) in the spectrum. ${ }^{20}$ A motor-driven stage is used to rotate the cell from outside the hutch, in order to shift the Bragg peaks of the diamonds as far as possible from the region of interest; the real-time observation of the transmission spectrum makes this adjustment relatively easy.

The absorption spectrum of $\mathrm{GaP}$ has been measured at the $K$ edge of gallium (10367 eV) using an optimized $\mathrm{Si}[111]$ crystal as energy dispersive optics. Less than 10 min were necessary to obtain a good quality spectrum.

The energy calibration was made by measuring the absorption spectrum of GaAs at room pressure, and by comparing this spectrum with data collected using a step-by-step scan.

Only spectra obtained on powder samples were analyzed for the following reason: the overall shape of the absorption curves is similar for powder and for single crystals. With single crystalline samples it is not possible to fill the whole experimental volume, whereas with powder this problem does not arise. If a part of the beam goes directly to the detector the white-line intensity is strongly reduced. Such an effect is also observed when holes are in the sample.

In the analysis of the spectra, the threshold energy is taken at the inflection point of the edge.

\section{RESULTS AND DISCUSSION}

The oscillatory part of the absorption cross section is given by

$$
\chi(k)=\frac{\mu-\mu_{0}}{\mu},
$$

where $\mu_{0}$ is the atomic absorption, and $\mu$ the measured cross section. In a one-electron, single-scattering approximation where the wave function associated with the photoelectron is a plane wave,

$$
\begin{aligned}
\chi(k)=\sum_{j} & \frac{N_{j}}{k R_{j}^{2}}\left|f_{j}(k, \pi)\right| \sin \left[2 \mathbf{k} \cdot \mathbf{R}_{j}+\Psi_{j}(k)\right] \\
& \times e^{-\sigma^{2} k^{2}} e^{-2 R_{j} / \lambda_{j}(k)},
\end{aligned}
$$

where $N_{j}$ is the number of neighbor atoms at the distance $R_{j},\left|f_{j}(k, \pi)\right|$ the backscattering amplitude, $\lambda_{j}(k)$ the electron mean free path, $\sigma_{j}^{2}$ the mean-square displacement between the absorbing atom and the $j$ th backscattering atom (Debye-Waller factor), and $\Psi_{j}(k)$ the total phase shift due to the backscattering and the absorbing atom. The photoelectron wave vector $k$ is given by

$$
k=\hbar^{-1}\left[2 m\left(E-E_{0}\right)\right]^{1 / 2},
$$

where $E$ is the energy of the incident photon, and $E_{0}$ the threshold energy.

The above expressions imply that the photon extracts the photoelectron from the core level of the central atom (gallium in the present case). The wave associated with this photoelectron is then partly backscattered by the neighboring atoms, so that the final state of the dipolar transition is essentially a superposition of the outgoing wave and the backscattered wavelets, which give rise to the observed interferences. The plane-wave approximation is not essential, but it is given here to simplify the presentation. In the actual analysis, phase shifts are extracted from GaP at ambient pressure and therefore include the spherical aspect of the photoelectronic wave. At low energy, the mean free path of the electrons is large. Hence, a low-energy electron may be scattered by several neighbors, keeping an appreciable scattering amplitude, which includes multiple-scattering events: ${ }^{21}$ it is the XANES region ( $\mathrm{x}$-ray absorption near-edge structure), limited to approximately $50 \mathrm{eV}$ above the absorption edge, while the EXAFS part is above this limit.

The $\chi(k)$ is deduced from the experimental absorption spectrum by subtracting the base line, using a smoothing procedure. A Fourier transform of $\chi(k)$ is made in order to deduce information in real space. For every pressure, we used the same window for the Fourier-transform procedure.

In order to determine the nearest-neighbor interatomic distance $R$ and the variation of the Debye-Waller factor, the first step is to determine the backscattering amplitude $\left|f_{j}(k, \pi)\right|$ and the phase shift $\Psi_{j}(k)$ of the Ga-P pairs. This was done by measuring the EXAFS spectrum of $\mathrm{GaP}$ at ambient pressure; the structure and the distance between the various neighbors are known and the Debye-Waller factor is taken to be 0 . Since a determination of the absolute value of the Debye-Waller factor is not possible, only its pressure dependence can be obtained. $\left|f_{j}(k)\right|$ and $\Psi_{j}(k)$ are then adjusted in order to fit $\chi(k)$ calculated using Eq. (1) to the inverse Fourier transform (IFT) of the first-neighbor peak. 
The second step is to fit the $\chi(k)$ calculated using $\left|f_{j}(k)\right|$ and $\Psi_{j}(k)$ to the IFT of the first-neighbor peak, with two adjustable parameters $R$ and $\sigma$.

Using the procedure described, we have obtained the equation of state (EOS) of GaP up to $24 \mathrm{GPa}$ (i.e., just below the zinc-blende to tetragonal phase transition) (Fig. 2 ). In this figure, the points represent the $\mathrm{Ga}$ to $\mathbf{P}$ distance deduced from the EXAFS spectra, and the dashed line is a Murnaghan first-order EOS fitted on the results with two adjustable parameters, $B_{0}$, the bulk modulus at $P=0$, and $B_{0}^{\prime}$, its pressure derivative. For a cubic crystal, the Murnaghan EOS is written

$$
d=d_{0}\left(1+\frac{B^{\prime} P}{B_{0}}\right)^{-\left(1 / 3 B^{\prime}\right)}
$$

The best fit yields $B_{0}=87.4 \mathrm{GPa}$ and $B^{\prime}=4.5 \mathrm{GPa}$ using $d_{0}=2.37 \AA$. These results are in good agreement with ultrasonic results measured up to $0.15 \mathrm{GPa}$ (Ref. 6) $\left(B_{0}=88.19 \mathrm{GPa}\right.$ and $B^{\prime}=4.78 \mathrm{GPa}$ ). This is the first high-pressure measurement of the EOS of GaP.

At higher pressure, GaP undergoes a structural phase transition to a tetragonal metallic phase. ${ }^{12-14}$ This transition is clearly seen on the absorption spectrum, especially in the XANES range (Fig. 3). This figure shows the XANES spectrum of a sample at four pressures (evolution is from the top to the bottom). The first two spectra (1.5 and $11.5 \mathrm{GPa}$ ) are those of the zinc-blende phase where the gallium atom is surrounded by four phosphorus atoms: the spectrum is characterized by three low-energy peaks, the "white line" and two additional peaks. Such a feature is generally observed for tetrahedrally bound compounds. When the pressure is increased, these two peaks move towards higher energies, which reflects the shortening of the interatomic distances. ${ }^{25}$

More striking is the change of the shape of the XANES spectrum when the phase transition occurs. The third spectrum taken at the highest pressure reached in this experiment $(36 \mathrm{GPa})$ is definitely different from the

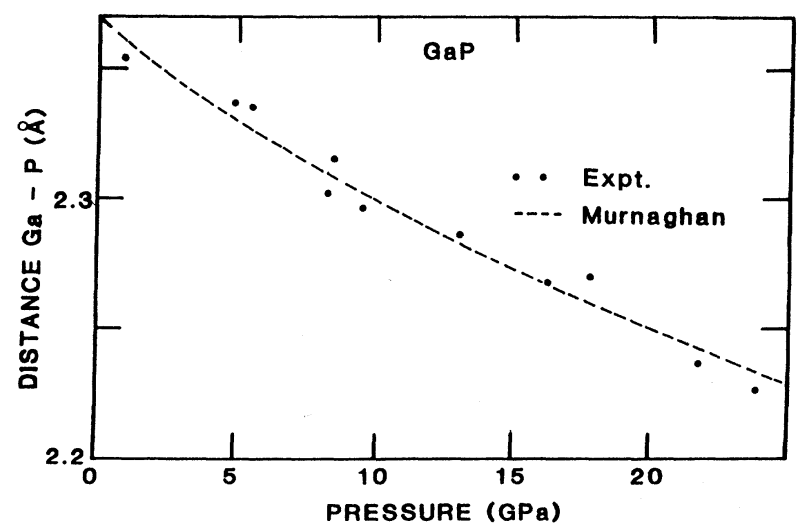

FIG. 2. First-neighbors interatomic distance in $\mathrm{GaP}$ as a function of pressure. The points represent the experimental results deduced from EXAFS analysis and the dashed line is the best-fit Murnaghan equation of state [Eq. (3)].

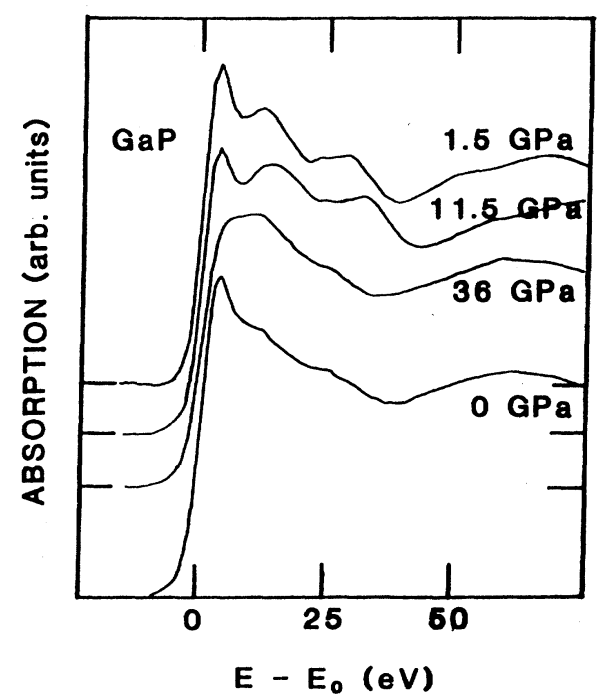

FIG. 3. XANES part of the spectrum at various pressures. The spectrum at $p=0 \mathrm{GPa}$ has been obtained after a rapid decrease of the pressure from $36 \mathrm{GPa}$.

low-pressure spectra. The structures characteristic of the tetrahedral environment disappear. Correlatively, there is a great simplification in the observed EXAFS signal, which is good enough to deduce information on the first shell of neighbors only.

The last spectrum obtained at $P=0$ in the same run (i.e., after quick relaxing the $36 \mathrm{GPa}$ pressure) is different from those in the low- and in the high-pressure phases: the white line is present, but the two well-defined peaks observed in phase I have disappeared.

Using the procedure described above, one can extract the EXAFS oscillations from the absorption spectra and make the Fourier transform of $\chi(k)$. The amplitudes of the Fourier transform of the spectra shown in Fig. 3 are presented in Fig. 4, where curve (a) is taken at 1.5, (b) at 11.5 , (c) at 36 , and (d) at $0 \mathrm{GPa}$ after releasing the pressure. In this figure the more intense peak is due to the first-neighbor shell. Curves 4(a) and 4(b) exhibit also at larger distance contributions due to further neighbors. These contributions do not appear clearly at $36 \mathrm{GPa}$ [curve 4(c)] and in the curve [curve 4(d)] for pressure released at 0 . As expected, the position of the firstneighbor peak shifts toward shorter distances during the initial pressure increase from 1.5 to $11.5 \mathrm{GPa}$. Normally the crystallographic distance is not directly given by the position of the IFT peak because of the $k$ dependence of the phase shift. Since there is no significant pressure dependence of the phase shift, the peak shift gives a direct measure of the pressure dependence of the Ga-P distance. When pressure is further increased to $36 \mathrm{GPa}$, the first-neighbor peak shifts toward larger distances: this phenomenon does not contradict the general trend of a pressure-induced densification. Actually the coordination number is 4 in the zinc-blende phase and jumps to 6 (4 $\mathrm{P}$ and $2 \mathrm{Ga}$ ) in the tetragonal high-pressure phase, giving rise to a higher density with a larger interatomic dis- 
tance for the first neighbors.

Quantitative information is derived by fitting the IFT of the first-neighbor peak. Three examples are shown in Fig. 5, where the IFT is shown by the solid line and the calculated spectrum by the dots. At $4.9 \mathrm{GPa}$, i.e., in phase I (zinc blende), the fit is performed with four first neighbors. The fit is excellent. In the tetragonal highpressure phase there is a good quality fit by using six first neighbors: four phosphorus and two gallium atoms. This fit is performed considering the two kinds of atoms and utilizing the phase shift and the amplitude of normal $\mathrm{GaP}$ for the phosphorus neighbors. We have a peculiar situation, due to the $P$ and $\mathrm{Ga}$ atoms being first neighbors together. In the considered energy range, the backscattering amplitude of gallium is weak and the phase of the oscillation due to this atom is in opposition with $P$ atoms. Such a situation is observed in Ni-As (Ref. 26) or $\mathrm{Zn}-\mathrm{Al}$ (Ref. 27) alloys as well. In order to extract the backscattering amplitude and the phase shift of $\mathrm{Ga}$ atoms, we chose to use the backscattering amplitude and the phase shift of As as determined from GaAs, where the first-neighbor distances are well separated, which is not the case for gallium metal, for example. It should not introduce a great difference, because the two atoms are almost neighbors in the Periodic Table. At $36 \mathrm{GPa}$, the best fit is obtained with four phosphorus at $2.41 \AA$ from the central atom and two gallium at $2.44 \AA$.

The third spectrum of Fig. 5 shows the fit obtained on

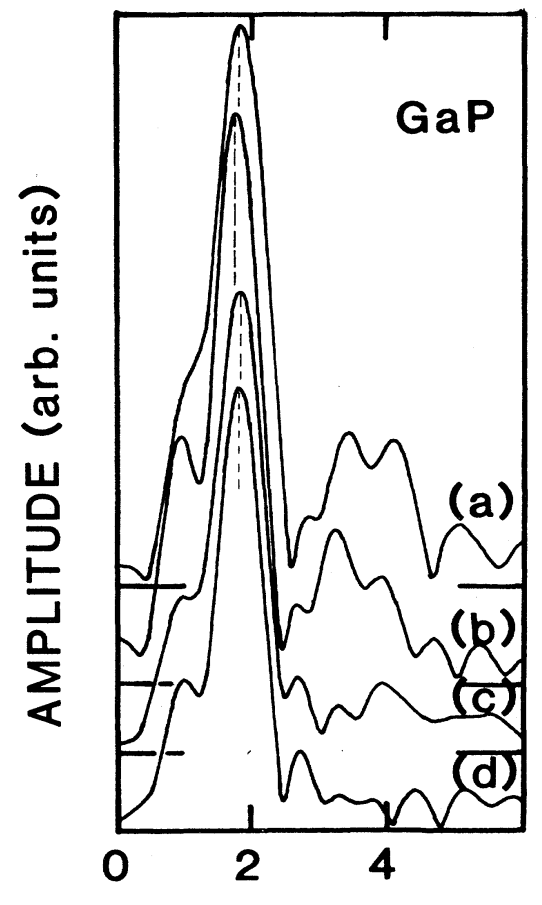

\section{DISTANCE $(\AA)$}

FIG. 4. Amplitude of the Fourier transform of the EXAFS oscillations at various pressures. (a), $1.5 \mathrm{GPa}$; (b), $11.5 \mathrm{GPa}$; (c), $36 \mathrm{GPa}$; (d), $0 \mathrm{GPa}$ after a rapid decrease from $36 \mathrm{GPa}$. the same sample after releasing the pressure. In this fit, the number of first neighbors and the distance were free parameters. The best fit is obtained with 3.1 phosphorus as first neighbors at a distance of $2.39 \AA$ (the nearestneighbor's distance in GaP phase I is $2.36 \AA$ ). The comparison of the absorption spectrum of the recovered sample with that of an amorphous GaP sample ${ }^{28}$ (Fig. 6) shows a great similarity. In the EXAFS range, the oscillations coincide, but in the XANES spectrum of the recovered sample, little of the ascendent crystalline structure remains, but it is heavily damped, resembling that of the amorphous sample. The conclusion from this comparison is that when pressurization is carried far above the phase-transition pressure, the recovered sample is almost entirely amorphous. Using $\mathrm{x}$-ray diffraction, $\mathrm{Hu}$ et al. ${ }^{14}$ did not find any evidence for amorphization on a sample recovered at room pressure after a pressurization up to $29.6 \mathrm{GPa}$. In fact, these authors obtained the x-ray

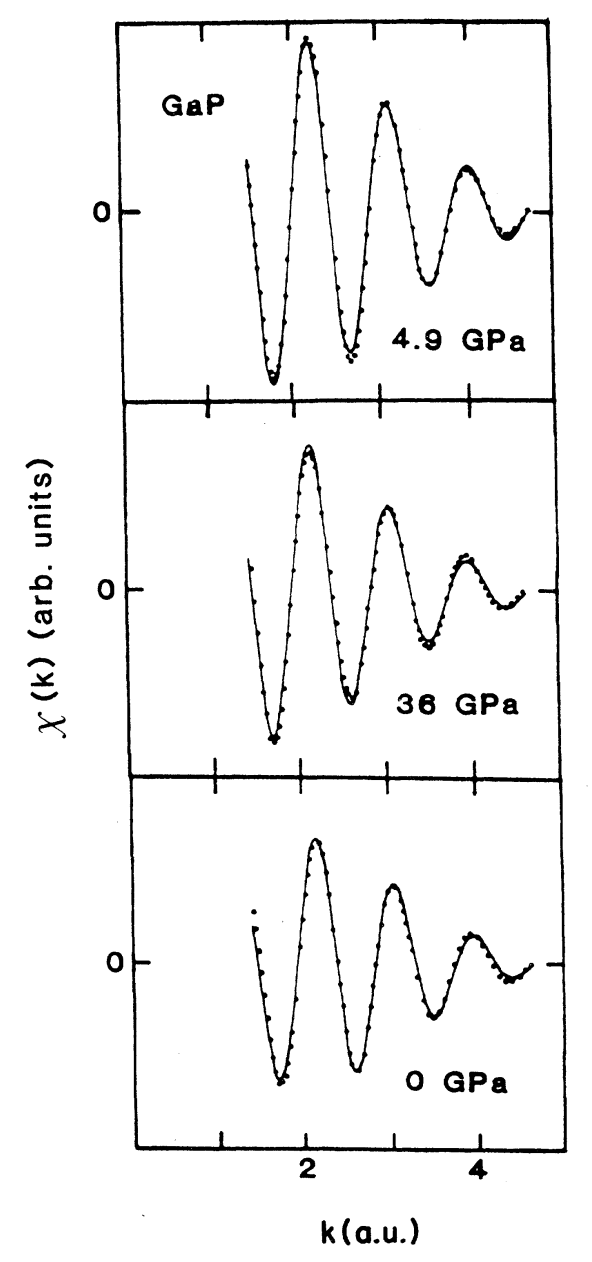

FIG. 5. First-neighbors filtered EXAFS oscillations at various pressures. The continuous line is the experimental spectrum and the dotted line is the fit using Eq. (1). $4.9 \mathrm{Gpa}$ : four phosphorus neighbors at $2.34 \AA$. $36 \mathrm{GPa}$ : four phosphorus neighbors at $2.41 \AA$ and two gallium neighbors at $2.44 \AA$. 0 GPa (recovered sample): 3.1 phosphorus neighbors at $2.39 \AA$. 


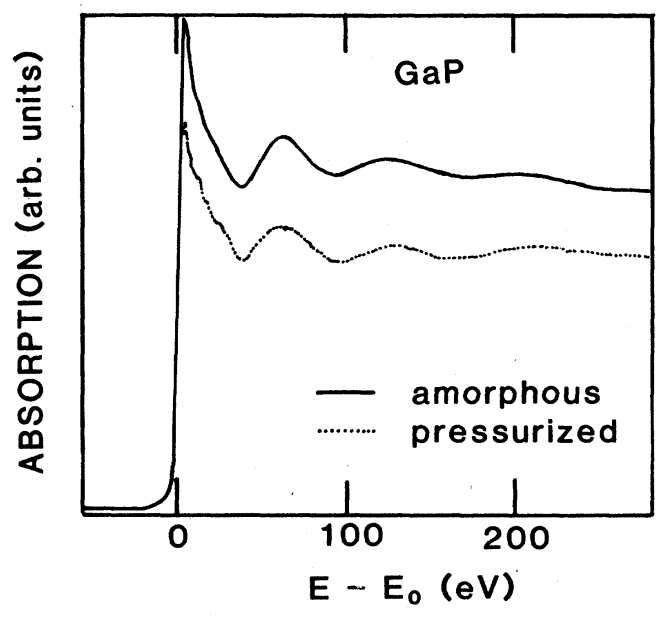

FIG. 6. Comparison of the EXAFS spectra of the recovered sample (dotted line) and an amorphous sample (Ref. 28) (solid line). The vertical scale is not the same in order to avoid the superposition of the curves.

pattern characteristic of phase I, except that all the peaks were weaker than in the original spectra of phase $I$. This change was attributed to an irreversible decrease of the thickness of the gasket (and therefore of the sample) when high pressure is applied. Nevertheless, it should be recalled that at $25 \mathrm{GPa}$ they found that only $75 \%$ of the sample was in the high-pressure phase. Since the pressure was not raised to a significantly much higher pressure $(29.6 \mathrm{GPa})$, it is likely that in their experiment the transition was not completed. In fact, this could explain why they found phase $I$ in their $x$-ray pattern of the recovered sample. A more recent zero-pressure electondiffraction study carried out on a sample pressurized to 28.4 GPa and x-ray-diffraction study on the sample pressurized to $32 \mathrm{GPa}$ show the presence of a mixture of amorphous and polycrystalline GaP. The transmission spectra on a sample quenched from $32.5 \mathrm{GPa}$ is similar to that of annealed amorphous GaP. ${ }^{8}$

\section{CONCLUSION}

GaP has been studied as a function of pressure in a diamond anvil cell by $\mathrm{x}$-ray-absorption spectroscopy up to $36 \mathrm{GPa}$. The analysis of the spectra shows the following. (i) It yields the equation of state in the zinc-blende phase up to the phase transition. (ii) The transition from phase I, where the gallium atoms are fourfold coordinated, to the $\beta$-tin structure, where it is sixfold coordinated, is clearly seen on the EXAFS and on the XANES part of the spectrum, which is very sensitive to a change in the local environment of the absorbing atom. (iii) The sample recovered at $p=0$ after pressurization to $36 \mathrm{GPa}$ is mainly amorphous. The long-range order of the recovered sample appears to be dependent on the maximum pressure reached.

\section{ACKNOWLEDGMENTS}

Physique des Milieux Condensés is Unité Associé no. 782 of Centre National de Recherche Scientifique. Laboratoire pour l' Utilisation du Rayonnement Électromagnétique (Orsay, France) is associated with Centre National de la Recherche Scientifique (Paris, France), Ministere de l'Education Nationale de la Jeunesse et des Sports (MENJS), and Commissariat à l'Energie Atomique. This work has been supported by MENJS under Contract No. 8850171.
${ }^{1}$ B. A. Weinstein, J. B. Renucci, and M. Cardona, Solid State Commun. 12, 473 (1973).

${ }^{2}$ B. A. Weinstein and G. J. Piermarini, Phys. Lett. 48A, 14 (1974).

${ }^{3}$ B. A. Weinstein and G. J. Piermarini, Phys. Rev. B 12, 1172 (1975).

${ }^{4}$ J. P. Pinceaux, J. M. Besson, A. Rimsky and G. Weill, in High Pressure in Science and Technology, edited by B. Vodar and Ph. Marteau (Pergamon, Oxford, 1980), p. 241.

${ }^{5}$ K. Aoki and M. Cardona, Phys. Status Solidi, B 122, 441 (1984).

${ }^{6}$ Y. K. Yog̃urtcu, A. J. Miller, and G. A. Saunders, J. Phys. Chem. Solids 42, 49 (1981).

${ }^{7}$ S. Ves, K. Strossner, C. K. Kim, and M. Cardona, Solid State Commun. 55, 327 (1985).

${ }^{8}$ C. Jauberthie-Carillon and C. Guillemin (unpublished).

${ }^{9}$ K. Strossner, S. Ves, and M. Cardona, Phys. Rev. B 32, 6614 (1985).

${ }^{10}$ F. P. Bundy, Rev. Sci. Instrum. 46, 1318 (1975).

${ }^{11}$ C. G. Homan, D. P. Kendall, T. E. Davidson, and J. Frankel, Solid State Commun. 17, 831 (1975).

${ }^{12}$ S. C. Yu, I. L. Spain, and E. F. Skelton, Solid State Commun. 25, 49 (1978).
${ }^{13}$ M. Baublitz, Jr. and A. L. Ruoff, J. Appl. Phys. 63, 6179 (1982).

${ }^{14}$ J. Z. Hu, D. R. Black, and I. L. Spain, Solid State Commun. 51, 285 (1984).

${ }^{15}$ R. Ingalls, G. A. Garcia, and E. A. Stern, Phys. Rev. Lett. 40, 334 (1978).

${ }^{16}$ R. Ingalls, E. D. Crozier, J. E. Whitmore, A. J. Seary, and J. M. Tranquada, J. Appl. Phys. 51, 3158 (1980).

${ }^{17}$ J. P. Itie, M. Jean-Louis, E. Dartyge, A. Fontaine, and A. Jucha, EXAFS and Near-Edge Structure IV [J. Phys. (Paris) Colloq. 47, C8-897 (1986)].

${ }^{18}$ R. Munch, H. D. Hochheimer, A. Werner, G. Materlik, A. Jayaraman, and K. V. Rao, Phys. Rev. Lett. 50, 1619 (1983).

${ }^{19}$ J. Rohler, J. P. Kappler, and G. Krill, Nucl. Instrum. Methods 208, 647 (1983).

${ }^{20}$ A. Polian, J. P. Itie, E. Dartyge, A. Fontaine, and G. Tourillon, Phys. Rev. B 39, 3369 (1989).

${ }^{21}$ E. Dartyge, C. Depautex, J. M. Dubuisson, A. Fontaine, J. Jucha, and G. Tourillon, Nucl. Instrum. Methods A 246, 452 (1986).

${ }^{22}$ H. Tolentino, E. Dartyge, A. Fontaine, and G. Tourillon, J. Appl. Crystallogr. 21, 15 (1988).

${ }^{23}$ G. J. Piermarini, S. Block, J. D. Barnett, and R. A. Forman, J. 
Appl. Phys. 46, 2774 (1975).

${ }^{24}$ H. K. Mao, P. M. Bell, J. W. Shaner, and D. J. Steinberg, J. Appl. Phys. 49, 3276 (1978).

${ }^{25}$ M. Benfatto, C. R. Natoli, J. Garcia, and A. Bianconi, in EXAFS and Near-Edge Structure, edited by P. Lagarde, $D$. Raoux, and J. Petiau [J. Phys. (Paris) Colloq. 47, C8-25 (1986)].
${ }^{26} \mathrm{~S}$. Comin, in EXAFS and Near-Edge Structure III, edited by K. O. Hodgson, B. Hedman, and J. E. Penner-Hahn (Springer-Verlag, Berlin, 1984), p. 238.

${ }^{27}$ J. Mimault, A. Fontaine, P. Lagarde, D. Raoux, A. Sadoc, and D. Spanjaard, J. Phys. F 11, 1311 (1981).

${ }^{28}$ D. Udron, A. M. Flanck, A. Gheorghiu, P. Lagarde, and M. L. Theye, Philos. Mag. 59, 9 (1989). 